\title{
An experimental search strategy retrieves more precise results than PubMed and Google for questions about medical interventions
}

Robert G Badgett, Daniel P Dylla, Susan D Megison, E Glynn Harmon

Objective: We compared the precision of a search strategy designed specifically to retrieve randomized controlled trials (RCTs) and systematic reviews of RCTs with search strategies designed for broader purposes. Methods: We designed an experimental search strategy that automatically revised searches up to five times by using increasingly restrictive queries as long at least 50 citations were retrieved. We compared the ability of the experimental and alternative strategies to retrieve studies relevant to 312 test questions. The primary outcome, search precision, was defined for each strategy as the proportion of relevant, high quality citations among the first 50 citations retrieved.

Results: The experimental strategy had the highest median precision (5.5\%; interquartile range [IQR]: $0 \%$ - 12\%) followed by the narrow strategy of the PubMed Clinical Queries (4.0\%; IQR: 0\% - 10\%). The experimental strategy found the most high quality citations (median 2; IQR: 0 - 6) and was the strategy most likely to find at least one high quality citation (73\% of searches; $95 \%$ confidence interval $68 \%-78 \%$ ). All comparisons were statistically significant. Conclusions: The experimental strategy performed the best in all outcomes although all strategies had low precision. 


\section{An experimental search strategy retrieves more precise results than PubMed}

3 and Google for questions about medical interventions

4

5 Robert G. Badgett, MD

6 Department of Internal Medicine

7 Kansas University Medical Center - Wichita

81010 N. Kansas

9 Wichita, KS 67214-3199

10 rbadgett@kumc.edu

12 Authors' Present Affiliations:

13 Daniel P. Dylla, MSIS

14 Katy Campus Library

15 Houston Community College NW

16 Houston, TX 77084

17 Daniel.Dylla@hccs.edu

18

19 Susan D. Megison, MSIS

20 No institutional affiliation

21 Birmingham, AL

22 susan.megison@gmail.com

23

24 E. Glynn Harmon, Ph.D. (deceased)

25 School of Information, SZB 562D

26 University of Texas at Austin

27 Austin, TX 78712-0390 
29 Objective: We compared the precision of a search strategy designed specifically to retrieve randomized 30 controlled trials (RCTs) and systematic reviews of RCTs with search strategies designed for broader purposes.

31 Methods: We designed an experimental search strategy that automatically revised searches up to five times by 32 using increasingly restrictive queries as long at least 50 citations were retrieved. We compared the ability of the 33 experimental and alternative strategies to retrieve studies relevant to 312 test questions. The primary outcome, 34 search precision, was defined for each strategy as the proportion of relevant, high quality citations among the 35 first 50 citations retrieved.

36 Results: The experimental strategy had the highest median precision (5.5\%; interquartile range [IQR]: $0 \%$ 37 12\%) followed by the narrow strategy of the PubMed Clinical Queries (4.0\%; IQR: 0\% - 10\%). The 38 experimental strategy found the most high quality citations (median 2; IQR: 0 - 6) and was the strategy most 39 likely to find at least one high quality citation ( $73 \%$ of searches; $95 \%$ confidence interval $68 \%-78 \%$ ). All 40 comparisons were statistically significant.

41 Conclusions: The experimental strategy performed the best in all outcomes although all strategies had low 42 precision. 


\section{Introduction}

48 Health care providers are encouraged to answer clinical questions by first consulting evidence-based

49 summaries.(DiCenso, Bayley, \& Haynes, 2009) Summaries are defined as evidence-based practice guidelines

50 and evidence-based textbooks. (DiCenso, Bayley, \& Haynes, 2009) Accordingly, physicians commonly use

51 online resources such as UpToDate.(Anonymous, 2014; Edson et al., 2010; Duran-Nelson et al., 2013)

53 Unfortunately, summaries may not always suffice. The evidence-based summaries UpToDate, Dynamed

54 (Anonymous, 2014b), FirstConsult (Anonymous, 2014), and ACP Smart Medicine (Anonymous, 2014a) have

55 less than $5 \%$ overlap in the studies cited, which implies no resource is comprehensive(Ketchum, Saleh, \& Jeong,

56 2011). Similarly, studies report that UpToDate and the National Guidelines Clearinghouse addressed less than

$5780 \%$ of questions by primary care physicians(Fenton \& Badgett, 2007) and hospital-based physicians.(Lucas et

58 al., 2004)

59

60 At times health care providers must search for original studies due to the deficiencies of secondary resources

61 such as those discussed above; however, practicing physicians tend to have difficulty answering clinical

62 questions by using electronic databases. This difficulty places physicians in the position of "knowing less than

63 has been proved." (Mulrow, 1994). In a recent study, only 13\% of searches by physicians led to changing

64 provisional answers to correct while $11 \%$ of searches led to changing provisional answers to incorrect.

65 (McKibbon \& Fridsma, 2006) Lucas found that 14\% of inpatients were judged to have their care improved after

66 physicians received unsolicited search results provided as part of a research study.(Lucas et al., 2004)

67

68 The best search method for supplementing evidence-based summaries is controversial and difficult to identify

69 due to the absence of a direct comparison of commonly used methods. The use of PubMed is encouraged by

70 medical leaders;(Anonymous, 2014b; AAMC-HHMI Scientific Foundation for Future Physicians Committee,

71 2009) however, physicians prefer the speed and simplicity of Google.(Anonymous, 2014a; Sim, Khong, \& Jiwa, 
72 2008; Thiele et al., 2010) These methods fundamentally differ in the bibliographic data that are searched and in

73 the sorting of search results. PubMed by default sorts results by date which may obscure a seminal article with

74 more recent results from minor journals. On the other hand, Google, which sorts articles by a mix of estimated

75 importance and relevance, ignores the dates of publication or revision of sources. Thus, Google may not

76 accurately represent critical timing of search results that contain an article from a major journal that was later

77 contradicted in a less impactful journal. (Ioannidis, 2005) The implications of these differences are not fully

78 known. Google launched Scholar in 2004 in order to improve access to academic publications. As compared to

79 PubMed, Scholar indexes the full text of many journals rather than just the citation and abstract, but does not use

80 MEDLINE's metadata such as the National Library of Medicine's Medical Subject Headings (MeSH) terms and

81 publication types. Like Google web search, Google Scholar by default sorts citations by a mix of estimated

82 importance and relevance. The retrieval algorithms and heuristics deployed by Google Scholar are propriety, not

83 described on the Scholar website, and not clearly discernible.(Anonymous, 2014)

85 In 1998, one of the authors (RGB) launched the experimental search engine SUMSearch, which includes

86 PubMed searches and is specifically designed for use in clinical medicine to supplement evidence-based

87 textbooks and practice guidelines. (Badgett, 1999) The current version of SUMSearch is available at

88 http://sumsearch.org. SUMSearch preserves the date sorting feature used by PubMed, but allows automated

89 revisions of searches in order to make older sentinel articles visible. Automatic revisions of searches may

90 address barriers health care providers experience while searching, such as designing search strategies and

91 "uncertainty about narrowing the search...when faced with an overwhelming body of knowledge."(Ely et al., 92 2002)

94 Our objective is to quantify and compare the ability of a search designed specifically for clinical medicine with 95 alternative strategies that are designed for broader purposes. We hypothesized that an experimental search 96 strategy designed specifically for clinical topics would outperform other strategies for retrieving articles about 97 medical interventions. 


\section{Materials \& Methods}

99 We compared five search strategies taken from four search engines for their ability to answer a collection of

100 clinical questions. In previous comparisons, SUMSearch and PubMed have performed better than Scholar;

101 (Haase et al., 2007; Freeman et al., 2009; Anders\& Evans, 2010) however, the current study is the first to

102 compare SUMSearch and PubMed to each other and to Google. While Google and Google Scholar were not

103 designed for clinical purposes, the frequency of their use by health care providers mandates assessment of their

104 ability.

\section{Source of clinical questions}

106 We used questions about from the Clinical Questions Collection of the National Library of Medicine.

107 (Anonymous, 2004; Ely et al., 1999; Ely et al., 2005) The complete collection consists of 4654 questions

108 collected from physicians in Iowa. For each question, personnel at the National Library of Medicine assigned

109 keywords that were almost always taken from the MeSH database.

111 From the collection we included questions about treatment of non-pregnant adults. This exclusion allowed us to

112 better monitor development of the project as our clinical expertise is internal medicine. We excluded questions

113 that also had a keyword assigned for diagnosis in order to ensure that the questions focused on treatment and so

114 were best answered with randomized controlled trials and meta-analyses of trials. We excluded questions whose

115 keywords duplicated the keywords of other questions. We included 312 questions after the above exclusions

116 (Figure 1).

\section{Query expansion}

119 Each question in the Questions Collection contains a median of 2 keywords, usually based on Medical Subject 120 Heading $(\mathrm{MeSH})$ terms. We linked these keywords with "AND". In addition, we replaced the word "neoplasms" 121 with "cancer" and inverted all keywords that contained commas. For example, "anemia, sickle cell" was inverted 122 to "sickle cell anemia." This inversion allows the search term to also perform well as a text word. The resulting 
123 search terms were submitted to the search engines without designation of a search field so that at PubMed's

124 Clinical Queries the terms were searched as both MeSH terms and text words. All searches were performed 125 between June and December of 2009.

\section{Search strategies}

127 The experimental search strategy was based on the PubMed component of a prior version of SUMSearch

128 federated search engine and could perform up to five iterations for each question. Details and examples of the

129 iterations used by the experimental strategy are included in Table 2. This strategy sought randomized controlled

130 trials and systematic reviews of trials. Each iteration was progressively more restrictive. The composition and

131 sequencing of the iterations was based on experience with SUMSearch. The strategy returned the results of the

132 last iteration that retrieved 50 or more citations. The rationale for restricting the numbers of citations is to

133 reproduce the behavior observed in searchers to typically scan a limited number of citations. (Blair D, 1980;

134 Islamaj Dogan et al., 2009) This limit has been called the futility point and occurs when searchers regard

135 reviewing additional citations as being beyond their respective time and manageability constraints. The

136 experimental search strategy imitated PubMed searching by querying Entrez's eSearch utility.(Sayers, 2013)

137 This utility has no user interface and is designed by the National Library of Medicine for external search engines

138 and other automated tools to efficiently query PubMed.

139 We included two strategies from PubMed's Clinical Queries that are publicly available

140 (http://www.ncbi.nlm.nih.gov/pubmed/clinical). We used the current Narrow and Broad strategies for therapies.

141 These strategies were initially developed by Haynes in 1994 and revised by Haynes in 2005. (Haynes et al.,

142 1994; Haynes et al., 2005)

143 We studied two strategies by Google. We used the main Google Web search engine and labeled this strategy as

144 "Google." We used the Google Scholar search engine and labeled this strategy as "Scholar". For both of these

145 strategies, we assessed methods to improve upon simply constructing search queries by using clinical terms.

146 Using test cases, we informally assessed the benefit of adding the following candidate search terms to the search 
147 query: "PMID", "DOI", random, trial, site:.org, site:.edu, and site:.gov. The terms PMID and DOI are

148 abbreviations for "PubMed identifier" and "digital object identifier" and are common numeric identifiers in the

149 Internet addresses and on the Internet pages for articles in health care journals. These identifiers are indexed by

150 Google like any other content on an Internet page or in its Internet address. In addition, formal citations to health 151 care articles, such as in wikis, frequently include these numbers and the abbreviations that indicate the type of 152 number. The final strategy chosen for both Google and Scholar appended the strings "PMID”, “ $\sim$ random", and

153 " trial" to the search terms. The " " character was required at the time of our study for Google to seek

154 synonyms for an adjacent search term.(Schwartz, 2013) We appended "num=50" to the urls submitted for both 155 strategies in order to retrieve 50 hits per search. Searches were performed on a dedicated server that had Google 156 cookies removed in order to prevent Google from any customization of search results such as prioritizing results 157 based on geographic location.

\section{Outcome ascertainment}

159 All search results were parsed for PMIDs and DOIs. For search results from Google, we also parsed the text in 160 the Internet addresses of hyperlinks. All identifiers found were then submitted to Entrez's efetch utility in order 161 to retrieve full citations including PMIDs, MeSH terms and lists of all articles that commented on the retrieved 162 articles.

\section{Reference standard}

164 The reference standard required articles to be relevant and high quality. An article was considered relevant to the 165 clinical question if the article contained all of the keywords assigned by the Clinical Questions Collection to the 166 clinical question. The keywords could be either MeSH terms or MeSH entry terms, and the keywords could be 167 located in title, abstract, or MeSH terms of the article.

168 An article was considered high quality if it had high quality methodology or was considered important by an 169 expert in the domain of the article. Articles having high methodological standards were considered those that 
170 were reviewed by an evidence-based synoptic journal as previously done by

171 Aphinyanaphongs.(Aphinyanaphongs et al., 2005) These journals were ACP Journal Club, InfoPoems, Evidence

172 Based Dentistry, Evidence Based Medicine, Evidence Based Nursing, and Evidence Based Mental Health.

173 Articles considered important by a domain expert were those that were published with an accompanying 174 editorial.

175 To avoid incorporation bias that would artificially inflate our estimated of the accuracy of the searches, all 176 strategies were designed without incorporating search terms that contribute to the definition of the reference 177 standard. For example, one component of our reference standard is abstraction of the article by the publication 178 ACP Journal Club. Some websites, such as PubMed, indicate which citations have been reviewed by ACP 179 Journal Club. Thus, we could have added "ACP Journal Club" to our search strategy to improve its precision. 180 However, we did not add this term, as it would create incorporation bias and limit the ability to generalize the 181 results of our study to topics not covered by ACP Journal Club. An example question from the Clinical 182 Questions Collection and the resulting search strategies is in Table 1

\section{Statistical analysis}

184 The primary outcome was the median average precision of the searches for retrieving studies meeting criteria for 185 the reference standard. We limited the number of search results examined to 50 to control for the varying 186 number of results retrieved by each search engine. For example, searches for medical interventions may retrieve 187 hundreds of thousands of results using the Google strategy while retrieving a much smaller number with the 188 other search strategies. We specified 50 search results because searchers, outside of those performing meta189 analysis, are unlikely to review a large number of citations.(Blair D, 1980; Islamaj Dogan et al., 2009) In 190 addition, this limit allows comparison of searches that may retrieve substantially different number of citations.

191 (Herskovic, Iyengar, \& Bernstam, 2007) For example, Google may retrieve more citations of high quality than 192 the other strategies due to retrieving many-fold more total citations. However, the Google search is not clearly 193 better because the user had to sift through more citations to find the high quality citations. 
194 The precision was calculated as the proportion of the first 50 search results identified by each strategy that were

195 deemed to be relevant, high quality studies according the criteria in the preceding section, "Reference standard."

196 If no qualifying studies were retrieved, the precision was set to 0.

197 The number need to read (NNR) for each strategy is the number of citations that would have to be assessed to 198 yield one qualifying article. The NNR was calculated as the inverse of the precision. (Toth, Gray, \& Brice, 2005)

199 Calculations were made with R statistical software package, version 2.11.1.(R Development Core Team, 2012)

200 Pairwise comparisons between individual medians were assessed using a post hoc analysis for Friedman's Test.

201 Rates of dichotomous outcomes were compared with the chi-square test. Chi-square is a conservative choice as

202 it does not consider pairing of data in calculation.

\section{Results}

204 The most common clinical concepts in the 312 questions about treating non-pregnant adults were hyperlipidemia 205 (15 questions), hypertension (10 questions), and urinary tract infections (10 questions).

207 The principal outcome, search precision, and all other outcomes were not normally distributed (Lilliefors 208 normality test $\mathrm{p}<0.001$ ), so the median precision became the principal outcome. Using Google as an example to 209 illustrate the results, when the first 50 hits in a Google search were examined, a mean of 23 PubMed citations 210 were retrieved by parsing PMIDs or DOIs from the Google results (not shown in table). Of these 23 PubMed

211 citations, an average of 3.3 were deemed high quality because the citation was abstracted by an evidence-based 212 synoptic journal or published with an accompanying editorial. Of the 3.3 citations, an average of 1.3 was

213 relevant to the original search terms. While this suggests the mean precision for Google was 1.3 divided by 23 , 214 or $5.6 \%$, the actual mean precision was lower at $4.6 \%$. The discrepancy is because the average of a series of 215 fractions is not equivalent to the average of the numerators divided by the average of the denominators. Lastly, $21654 \%$ searches performed by Google retrieved no high quality, relevant citations thus the median precision for 217 Google was $0 \%$ (Table 3). The corresponding values for the numbers needed to read are: Experimental 18, 
218 PubMed narrow Clinical Query 25, and PubMed's broad Clinical Query 50. The numbers needed to read cannot

219 be calculated for the Google strategies due to their median precision of $0 \%$.

220 The median precision was significantly different among the strategies by Friedman's rank sum test (Table 3).

221 The experimental strategy and the narrow strategy of the PubMed Clinical Queries had the highest median

222 precision (5.5\% and 4.0\%, respectively). The experimental strategy had the highest ranked and mean values of

223 precision (Table 3; $\mathrm{p}<0.001$ for both analyses). The experimental strategy was the most likely method to find at

224 least one high quality citation ( $73 \%$ of questions) with $\mathrm{p}<0.001$. The median number of high quality articles

225 retrieved per search was two for both the experimental strategy and the PubMed narrow, while the means were

2265.0 and 2.6 , respectively $(\mathrm{p}<0.001)$.

227 In an unplanned analysis, we examined the precision of experimental search strategies based on the number of

228 iterations the experimental strategy required (Figure 2). Searches that required one or two iterations had low

229 precision, whereas searches requiring more iterations had higher precision.

230 For all outcomes, Google and Google Scholar performed worse than the other strategies. This was in part

231 because Google itself sometimes found high quality citations that were not relevant. For example, in a search for

232 bronchiectasis and drug therapy, Google retrieved the Wikipedia pages on acetylcysteine and pulmonary

233 embolism. The acetylcysteine page was retrieved because Wikipedia listed bronchiectasis as treatable with

234 acetylcysteine while the pulmonary embolism page was retrieved only because the page listed bronchiectasis in

235 the page's navigational menu of pulmonary diseases. Unfortunately, the high quality citations that were on these

236 pages were not relevant to bronchiectasis.

\section{Discussion}

238 The experimental search was significantly better than the other strategies in all outcomes. Google and Google

239 Scholar strategies did not perform as well. We believe this is the first comparative study to identify a search

240 strategy that may be comparable to or better than the 2004 version of the PubMed Clinical Queries for common 
241 clinical questions. The experimental search is available at http://sumsearch.org/ by changing the default settings

242 so that "Focus" is Intervention, "Target \# of original studies" is 50, and "Require abstracts" is not selected.

244 Our results support Battelle's hypothesis that domain-specific search strategies should perform better than 245 general strategies.(Battelle, 2005) Google and Google Scholar's poor performance was consistent with prior 246 comparisons with PubMed or SUMSearch. (Haase et al., 2007; Freeman et al., 2009; Anders\& Evans, 2010) Our 247 study should be compared to three studies that suggest benefit from using Google Scholar. Gehanno notes 248 perfect coverage by Scholar of trials in a set of Cochrane reviews. (Gehanno, Rollin, \& Darmoni, 2013)

249 However, coverage simply relates to the presence of trials in the Scholar database, which is different from our 250 study of how well those trials can be retrieved by search strategies. Two smaller studies, by Nourbakhsh and 251 Shariff suggests that Scholar retrieves more citations that are relevant than PubMed retrieves. (Nourbakhsh et 252 al., 2012; Shariff et al., 2013) Several reasons may underlie the conflicting results. The reference standard used 253 by Nourbakhsh only considered relevance and not study design or quality of citations. Our precision is likely 254 underestimated due to the certain existence of qualifying articles that were missed due to not being abstracted by 255 an evidence based synoptic journal. Also, the PubMed searches used by Nourbakhsh relied exclusively on 256 MeSH terms. (Nourbakhsh et al., 2012) For example, Nourbakhsh used "hypertension, pulmonary [MeSH]" 257 whereas we would have changed this term to "pulmonary hypertension[all fields]". The 'all fields' tag submits 258 the term as both a MeSH term and a text word. In addition, the Nourbakhsh study was limited to four questions 259 the researchers were familiar with and the differences did not reach statistical significance. Shariff did not 260 provide details on how the nephrologists used PubMed other than stating that the searches were not revised 261 based on the number of results retrieved.(Shariff et al., 2013) The findings of similar precision of results yet 262 fewer relevant citations among the first 40 citations retrieved by PubMed compared to Scholar indicates that in 263 many cases the PubMed searches retrieved fewer than 40 citations. The conflict between our results and those of 264 Shariff may be due to our use of iterative searching or to the nature of primary versus specialty care questions.

265 Iterative searching may be more important in broad topics that retrieve more citations. 
267 The domain-specific search strategies that we studied, PubMed and SUMSearch, may perform better for two

268 reasons that have not changed since our study was completed. First, these strategies, unlike Google and Scholar,

269 take advantage of the hierarchal Medical Subject Headings (MeSH) terms that the National Library of Medicine

270 assigns to citations. Second, our results raise the question of whether a Boolean search model should be

271 preferred for the task we studied. Most contemporary research of searching MEDLINE examines search models

272 other than Boolean. Boolean models connect search terms with logical connectors such as 'and' and 'or' are

273 considered weaker than other search models. (Baeza-Yates \& Ribeiro-Neto, 2011) A paradoxical advantage of

274 Boolean models is that because they do not rank documents by any grading scale, search results can be sorted by

275 date of publication. Sorting by date can be critical in medicine because of the surprising frequency that research

276 results are contradicted by subsequent authors.(Ioannidis, 2005)

278 In addition to providing a comparison of the performances of commonly used search strategies, our results 279 reinforce the difficulty of retrieving clinical studies from MEDLINE. The experimental strategy was most 280 precise but barely achieved a precision of 5\%. Our study reported substantially lower precision than a previous 281 comprehensive review by McKibbon.(McKibbon et al., 2009) Common to our study and the review was analysis 282 of the PubMed Clinical Queries narrow filter. McKibbon reported a precision of 55\% whereas we found the 283 same filter to have a precision of $2 \%$. We believe our study reflects the precision that health care providers will 284 encounter and is lower than the report of McKibbon for two reasons. First, we measured the precision in 285 answering actual clinical questions. Second, we measured the precision among all journals of PubMed rather 286 than limiting to the 161 journals that publish the highest rate of high-quality studies. Since we executed our 287 study, Shariff reported that nephrologists were able to search MEDLINE with a mean precision higher than our 288 report of median precision. (Shariff et al., 2013) We reported median rather than mean values for precision due 289 to concern that means will overstate performance. To directly compare studies, the mean precision of $10.2 \%$ we 290 report for our experimental strategy is higher than found by Shariff.

\section{Possible limitations}


293 First, we standardized the design of all search strategies to eliminate variability in the search skill of actual users.

294 Both the precision and number of relevant citations retrieved by human searchers may be less than we report. It

295 is possible that in our study Google's performance was diminished because Google may have found citations

296 that were not counted because they were not accompanied by PMIDs or DOIs. However, in addition to parsing

297 the results displayed by Google, we also parsed the links provided by Google. Any functional link to an article at

298 PubMed will have a PMID embedded and be found by our methodology. Similarly, high quality studies may

299 have been missed by all strategies due to our removing "diagnosis" as a key word. This may have selectively

300 harmed the experimental and PubMed strategies as these incorporated MeSH terms. However, it is unknown

301 whether this affected precision as the total number of studies retrieved is also lower.

303 We recognize that our definition of the reference standard might be debatable for three reasons. First, we limited

304 our study to retrieving randomized controlled trials and systematic reviews of randomized controlled trials

305 because treatment questions are important and the standards for the conduct and assessment of these studies are

306 better developed than for other resources. While this information need may be infrequent for many health care

307 providers, we believe the ability to locate randomized controlled trials is very important for peer leaders who

308 may be writing or teaching clinical topics. Second, our definition of high quality articles is imperfect. We

309 believe, however, our definition has the advantage of being determined by experts who determined that an

310 editorial or synopsis was justified and who were not involved in the evaluation of the search strategies. In

311 addition, we believe the results that our definition yields are likely to move in parallel with other definitions of

312 high quality. Third, the use of precision (the proportion of relevant documents retrieved in the search) as a

313 metric instead of sensitivity (the ability of the system to detect all relevant documents) is debatable. However,

314 our goal is to create searches with precision for clinicians rather than comprehensive searches for meta-analysts.

315 For example, high sensitivity may be more useful for meta-analyses that require comprehensive results. High

316 precision may be more useful for time-sensitive tasks that require relevant documents quickly. Regardless, we

317 do provide the numbers of high quality citations retrieved which should correlate with the sensitivity of a

318 strategy for a given question. 
320 Our results should not be generalized beyond searching for studies of interventions. The randomized controlled

321 trial index term used by the National Library of Medicine‘s Medical Subject Headings (MeSH) is unusually

322 accurate whereas MeSH terms for other study designs may be less accurate. (Haynes et al., 2005) None of the

323 strategies we tested may be appropriate for the conduct of meta-analysis when very high recall or sensitivity of

324 searches is required. Lastly, our questions all had carefully assigned MeSH terms. Searchers not facile with

325 MeSH terms may have lesser results.

\section{Future research}

328 Future research could address the strategies that were studied and compare them to search strategies based on

329 alternative search models. Aside from the search strategies developed by Haynes for PubMed's Clinical Queries,

330 the strategies were not formally developed. For example, we appended Google and Scholar strategies with "

331 PMID random trial" based on several use cases, but perhaps other restrictions would have performed better.

332 However, Google's performance was so low that substantial improvement from revising search terms seems

333 unlikely. Google frequently revises its search algorithms. (Anonymous, 2011) Until Google makes a major

334 change, such as recognizing MeSH terms and the hierarchical relationship among them, the impact of lesser

335 revisions on searching for medical research is not known. Continual research of Google is warranted. Regarding

336 the experimental strategy, perhaps other iterations, sequences of iteration, and number of iterations would

337 improve the search results. In addition, Wilczynski recently described how to improve the precision of the

338 Haynes strategies by adding "not” terms to searches of MEDLINE (Wilczynski, McKibbon, \& Haynes, 2011)

339 Future research could compare our strategy to strategies based on machine learning or citation analysis. Lastly,

340 we hope that search engines in the future will provide more than a list of citations and will add indicators of

341 credibility to citations and display the conclusions in a way to allow users to quickly assess the concordance 
342 among conclusions. The former is currently done by SUMSearch by indicating which citations are accompanied

343 by editorials and reviews by synoptic publications. The latter is being developed by AskHermes.(Yu, 2014)

\section{Conclusion}

345 Our results suggest that when health care providers need to supplement evidence-based summaries by searching

346 for high quality randomized controlled trials and systematic reviews of randomized controlled trials, an

347 experimental strategy designed specifically for clinical care may be more appropriate than the more general

348 strategies deployed by Google and PubMed Clinical Queries. 


\section{References}

351

352

353

354

355

356

357

358

359 360

361

362 363

364

365

366

367

368

369

370

371

372

373

374

375

376

377

378

379

AAMC-HHMI Scientific Foundation for Future Physicians Committee. 2009. Scientific Foundations for Future Physicians.

Anders, M. E. and D. P. Evans. 2010. "Comparison of PubMed and Google Scholar Literature Searches." Respiratory Care 55 (5): 578-583.

Anonymous. "About Google Scholar " Google, http://scholar.google.com/intl/en/scholar/about.html(accessed May 5, 2014).

- - -. "About the IOWA Questions Collection(s)." National Library of Medicine, last modified September, 2004, http://clinques.nIm.nih.gov/About.jsp(accessed May 5, 2014).

- - - "ACP Smart Medicine." American College of Physicians, http://smartmedicine.acponline.org(accessed May 5, 2014).

--_. "DynaMed ${ }^{T M} . "$ EBSCO Publishing, http://dynamed.ebscohost.com/(accessed May 5, 2014).

- - - "Facts about Google and Competition ", http://www.google.com/competition/howgooglesearchworks.html(accessed 12/30/2013, 2013).

---. "FirstCONSULT." Elsevier B.V., http://www.firstconsult.com/(accessed May 5, 2014).

---. "UpToDate ${ }^{\oplus . " ~ W o l t e r s ~ K l u w e r ~ H e a l t h, ~ h t t p: / / u p t o d a t e . c o m(a c c e s s e d ~ M a y ~ 5, ~ 2014) . ~}$

---. 2011. "Friedman Rank Sum Test." Chap. June 3, 2011, In R: A Language and Environment for Statistical Computing. Vol. 2010, 1321. Vienna, Austria: R Foundation for Statistical Computing. http://cran.rproject.org/doc/manuals/fullrefman.pdf.

-_-. "Google." Google, http://www.google.com/(accessed May 5, 2014).

_-_. "PubMed." National Center for Biotechnology Information, http://pubmed.gov(accessed May 5, 2014).

Aphinyanaphongs, Y., I. Tsamardinos, A. Statnikov, D. Hardin, and C. F. Aliferis. 2005. "Text Categorization Models for High-Quality Article Retrieval in Internal Medicine." Journal of the American Medical Informatics Association : JAMIA 12 (2): 207-216.

Badgett, R. G. 1999. "How to Search for and Evaluate Medical Evidence." Seminars in Medical Practice 2 (3): 814. http://www.turner-white.com/smp/abstractSMP.php?PubCode=smp oct99 medevid.

Baeza-Yates, Ricardo and Berthier Ribeiro-Neto. 2011. "Modeling." In Modern Information Retrieval: The Concepts and Technology Behind Search. 2nd ed., 57-130. Boston, MA: Addison-Wesley Professional.

Battelle, John. 2005. "Perfect Search." In The Search: How Google and its Rivals Rewrote the Rules of Business and Transformed our Culture. 1st ed., 274-276. New York: Portfolio. 
Blair D. 1980. "Searching Biases in Large Interactive Document Retrieval Systems." J Am Soc Inf Sci 31: 271-77.

381

382

383

384

385

386

387

388

389

390

391

392

393 394

395

396

397

398

399

400

401

402

403

404

405

406

407

408

409

410

411

412

413

DiCenso, A., L. Bayley, and R. B. Haynes. 2009. "ACP Journal Club. Editorial: Accessing Preappraised Evidence: Fine-Tuning the $5 S$ Model into a 65 Model." Annals of Internal Medicine 151 (6): JC3-2, JC3-3. http://www.annals.org/content/151/6/JC3-2.citation.

Duran-Nelson, Alisa, Sophia Gladding, Jim Beattie, and L. James Nixon. 2013. "Should we Google it? Resource use by Internal Medicine Residents for Point-of-Care Clinical Decision Making." Acad Med Publish Ahead of Print (6). doi:10.1097/ACM.0b013e31828ffdb7.

Edson, R. S., T. J. Beckman, C. P. West, P. B. Aronowitz, R. G. Badgett, D. A. Feldstein, M. C. Henderson, J. C. Kolars, and F. S. McDonald. 2010. "A Multi-Institutional Survey of Internal Medicine Residents' Learning Habits." Medical Teacher 32 (9): 773-775.

Ely, J. W., J. A. Osheroff, M. L. Chambliss, M. H. Ebell, and M. E. Rosenbaum. 2005. "Answering Physicians' Clinical Questions: Obstacles and Potential Solutions." Journal of the American Medical Informatics Association : JAMIA 12 (2): 217-224. doi:M1608 [pii]; 10.1197/jamia.M1608 [doi].

Ely, J. W., J. A. Osheroff, M. H. Ebell, G. R. Bergus, B. T. Levy, M. L. Chambliss, and E. R. Evans. 1999. "Analysis of Questions Asked by Family Doctors regarding Patient Care." BMJ 319 (7206): 358-61.

Ely, J. W., J. A. Osheroff, M. H. Ebell, M. L. Chambliss, D. C. Vinson, J. J. Stevermer, and E. A. Pifer. 2002. "Obstacles to Answering Doctors' Questions about Patient Care with Evidence: Qualitative Study." BMJ 324 (7339): 710.

Fenton, S. H. and R. G. Badgett. 2007. "A Comparison of Primary Care Information Content in UpToDate and the National Guideline Clearinghouse " Journal of the Medical Library Association : JMLA 95 (3): 255-259.

Freeman, M. K., S. A. Lauderdale, M. G. Kendrach, and T. W. Woolley. 2009. "Google Scholar Versus PubMed in Locating Primary Literature to Answer Drug-Related Questions." The Annals of Pharmacotherapy 43 (3): 478-484. doi:10.1345/aph.1L223.

Gehanno, J. F., L. Rollin, and S. Darmoni. 2013. "Is the Coverage of Google Scholar enough to be used Alone for Systematic Reviews?" BMC Medical Informatics and Decision Making 13: 7-11. doi:10.1186/1472-694713-7; 10.1186/1472-6947-13-7.

Haase, A., M. Follmann, G. Skipka, and H. Kirchner. 2007. "Developing Search Strategies for Clinical Practice Guidelines in SUMSearch and Google Scholar and Assessing their Retrieval Performance." BMC Medical Research Methodology 7: 28.

Haynes, R. B., K. A. McKibbon, N. L. Wilczynski, S. D. Walter, S. R. Werre, and Hedges Team. 2005. "Optimal Search Strategies for Retrieving Scientifically Strong Studies of Treatment from Medline: Analytical Survey." BMJ (Clinical Research Ed.) 330 (7501): 1179. doi:10.1136/bmj.38446.498542.8F.

Haynes, R. B., N. Wilczynski, K. A. McKibbon, C. J. Walker, and J. C. Sinclair. 1994. "Developing Optimal Search Strategies for Detecting Clinically Sound Studies in MEDLINE." J Am Med Inform Assoc 1 (6): 447-58. 
Herskovic, J. R., M. S. Iyengar, and E. V. Bernstam. 2007. "Using Hit Curves to Compare Search Algorithm Performance." Journal of Biomedical Informatics 40 (2): 93-99. doi:10.1016/j.jbi.2005.12.007.

Ioannidis, J. P. 2005. "Contradicted and Initially Stronger Effects in Highly Cited Clinical Research." JAMA 294 (1538-3598): 218-228. doi:10.1001/jama.294.2.218.

Islamaj Dogan, R., G. C. Murray, A. Neveol, and Z. Lu. 2009. "Understanding PubMed User Search Behavior through Log Analysis " Database : The Journal of Biological Databases and Curation 2009: bap018. doi:10.1093/database/bap018.

Ketchum, A. M., A. A. Saleh, and K. Jeong. 2011. "Type of Evidence Behind Point-of-Care Clinical Information Products: A Bibliometric Analysis." Journal of Medical Internet Research 13 (1): e21.

Lucas, Brian P., Arthur T. Evans, Brendan M. Reilly, Yuri V. Khodakov, Kalyani Perumal, Louis G. Rohr, Joseph A. Akamah, Tunji M. Alausa, Christopher A. Smith, and Jeremy P. Smith. 2004. "The Impact of Evidence on Physicians' Inpatient Treatment Decisions." Journal of General Internal Medicine 19 (5p1): 402-409. doi:10.1111/j.1525-1497.2004.30306.x.

McKibbon, K. A. and D. B. Fridsma. 2006. "Effectiveness of Clinician-Selected Electronic Information Resources for Answering Primary Care Physicians' Information Needs." Journal of the American Medical Informatics Association : JAMIA 13 (6): 653-659. doi:10.1197/jamia.M2087.

McKibbon, K. A., N. L. Wilczynski, R. B. Haynes, and Hedges Team. 2009. "Retrieving Randomized Controlled Trials from Medline: A Comparison of 38 Published Search Filters." Health Information and Libraries Journal 26 (3): 187-202. doi:10.1111/j.1471-1842.2008.00827.x.

Mulrow, C. D. 1994. "Rationale for Systematic Reviews." BMJ (Clinical Research Ed.) 309 (6954): 597-599.

Nourbakhsh, E., R. Nugent, H. Wang, C. Cevik, and K. Nugent. 2012. "Medical Literature Searches: A Comparison of PubMed and Google Scholar " Health Information and Libraries Journal 29 (3): 214-222. doi:10.1111/j.1471-1842.2012.00992.x; 10.1111/j.1471-1842.2012.00992.x.

R Development Core Team. 2012. R: A Language and Environment for Statistical Computing. Vol. 2.15.1. Vienna, Austria: R Foundation for Statistical Computing.

Sayers, E. "E-Utilities Quick Start." National Center for Biotechnology Information, last modified December 14, 2011, http://www.ncbi.nlm.nih.gov/books/NBK25500/(accessed May 5, 2014).

Schwartz, B. "Google Drops another Search Operator: Tilde for Synonyms ", http://searchengineland.com/google-drops-another-search-operator-tilde-for-synonyms-

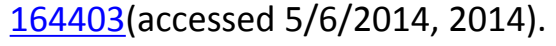

Shariff, S. Z., S. A. Bejaimal, J. M. Sontrop, A. V. Iansavichus, R. B. Haynes, M. A. Weir, and A. X. Garg. 2013. "Retrieving Clinical Evidence: A Comparison of PubMed and Google Scholar for Quick Clinical Searches." Journal of Medical Internet Research 15 (8): e164. doi:10.2196/jmir.2624; 10.2196/jmir.2624. 
447 Sim, M. G., E. Khong, and M. Jiwa. 2008. "Does General Practice Google? " Australian Family Physician 37 (6): $448 \quad 471-474$.

449 Thiele, R. H., N. C. Poiro, D. C. Scalzo, and E. C. Nemergut. 2010. "Speed, Accuracy, and Confidence in Google, 450 Ovid, PubMed, and UpToDate: Results of a Randomised Trial " Postgraduate Medical Journal 86 (1018): 451 459-465. doi:10.1136/pgmj.2010.098053.

452 Toth, B., J. A. Gray, and A. Brice. 2005. "The Number Needed to Read-a New Measure of Journal Value " Health 453 Information and Libraries Journal 22 (2): 81-82. doi:10.1111/j.1471-1842.2005.00568.x.

454 Wilczynski, N. L., K. A. McKibbon, and R. B. Haynes. 2011. "Search Filter Precision can be Improved by NOTing 455 Out Irrelevant Content " AMIA ...Annual Symposium Proceedings / AMIA Symposium.AMIA Symposium 456 2011: 1506-1513.

$457 \mathrm{Yu}, \mathrm{H}$. "AskHermes - the Clinical Question Answering System " University of Wisconsin-Milwaukee, $458 \quad$ http://www.askhermes.org/(accessed May 5, 2014). 


\section{Table $\mathbf{1}_{\text {(on next page) }}$}

Table 1. Example clinical question and resulting search strategy

* For users to reproduce the strategies with the current version of Google, settings are configured for "Google Instant Predictions" to be off and Results per page to be 50. The tilde signs are no longer required by Google as Google currently searches for synonyms by default. Since execution of our study, Google has revised Scholar to allow a maximum of 20 results per page. 
Table 1. Example clinical question and resulting search strategy (Clinical Questions Collection \# NQ001384).

\begin{tabular}{|l|l|}
\hline Original question by primary care physician & $\begin{array}{l}\text { "If someone had x-rays for acne treatment, how } \\
\text { should they be followed-up regarding thyroid } \\
\text { cancer risk?" }\end{array}$ \\
\hline Keywords originally assigned by the Clinical & Thyroid neoplasms \\
Questions Collection & Radiation Injuries \\
(Therapy category) and to Experimental search & Thyroid cancer AND Radiation Injuries \\
\hline Search submitted to Google and Scholar* & Thyroid cancer Radiation Injuries PMID $\sim$ random \\
& $\sim$ trial \\
\hline
\end{tabular}

* For users to reproduce the strategies with the current version of Google, settings are configured for "Google Instant Predictions" to be off and Results per page to be 50. The tilde signs are no longer required by Google as Google currently searches for synonyms by default. Since execution of our study, Google has revised Scholar to allow a maximum of 20 results per page. 
1

Selection of questions 


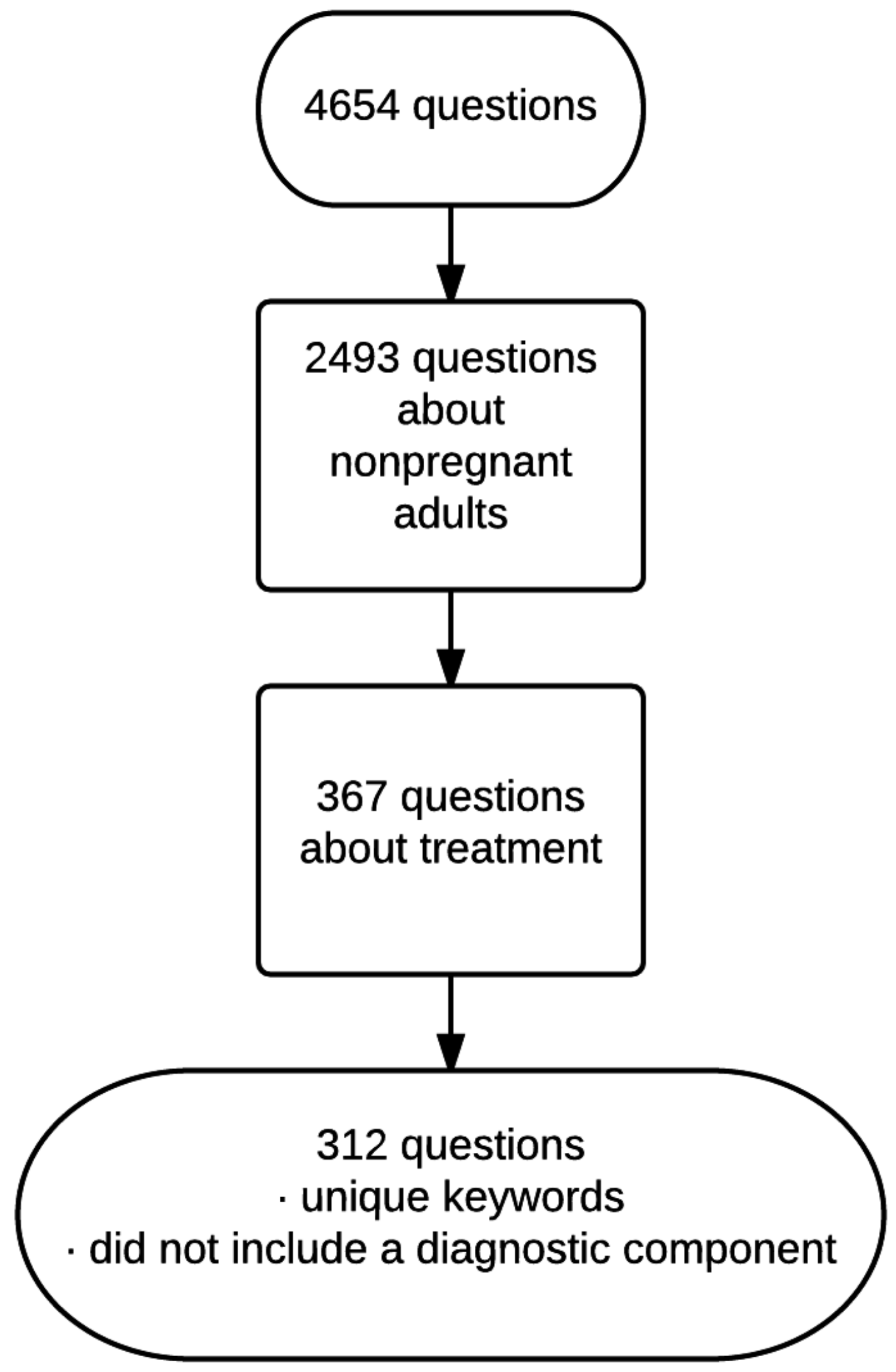




\section{Table 2 (on next page)}

Table 2. MEDLINE iterations of the experimental search strategy

* Filters are detailed in the original study by Haynes(Haynes et al. , 2005) and at http://hiru.mcmaster.ca/hiru/HIRU_Hedges_home.aspx. † Journals are listed at http://hiru.mcmaster.ca/hiru/hedges/MedlinejournalsRead.pdf. ‡ Medical Subject Headings terms assigned by the National Library of Medicine. 
Table 2. MEDLINE iterations of the experimental search strategy.

\begin{tabular}{|c|c|c|c|}
\hline \multirow{2}{*}{ Iteration } & \multicolumn{3}{|c|}{ Options to increase specificity of search } \\
\hline & Quality filters & Publication types & Additional query expansion \\
\hline 1 & No filter & None & None \\
\hline 2 & Haynes 2005 sensitive filter* or & Excluded publication & Required abstract \\
\hline & systematic review subset & type of review, letter, & \\
\hline 3 & Switched to Haynes 2005 specific & $\begin{array}{l}\text { editorial } \\
\text { No change }\end{array}$ & No change \\
\hline 4 & $\begin{array}{l}\text { filter or systematic review } * \\
\text { Added restriction to } 106 \text { journals }\end{array}$ & No change & No change \\
\hline 5 & $\begin{array}{l}\text { in McMaster list as of } 02 / 2008 \dagger \\
\text { No change }\end{array}$ & No change & Added restriction of \\
\hline & & & search terms to MeSH $\neq$ \\
\hline & & & major field \\
\hline
\end{tabular}

* Filters are detailed in the original study by Haynes(Haynes et al. , 2005) and at http://hiru.mcmaster.ca/hiru/HIRU_Hedges home.aspx.

$\dagger$ Journals are listed at http://hiru.mcmaster.ca/hiru/hedges/MedlineJournalsRead.pdf. $\ddagger$ Medical Subject Headings terms assigned by the National Library of Medicine. 
2

Precision by number of interations used by the experimental search engine

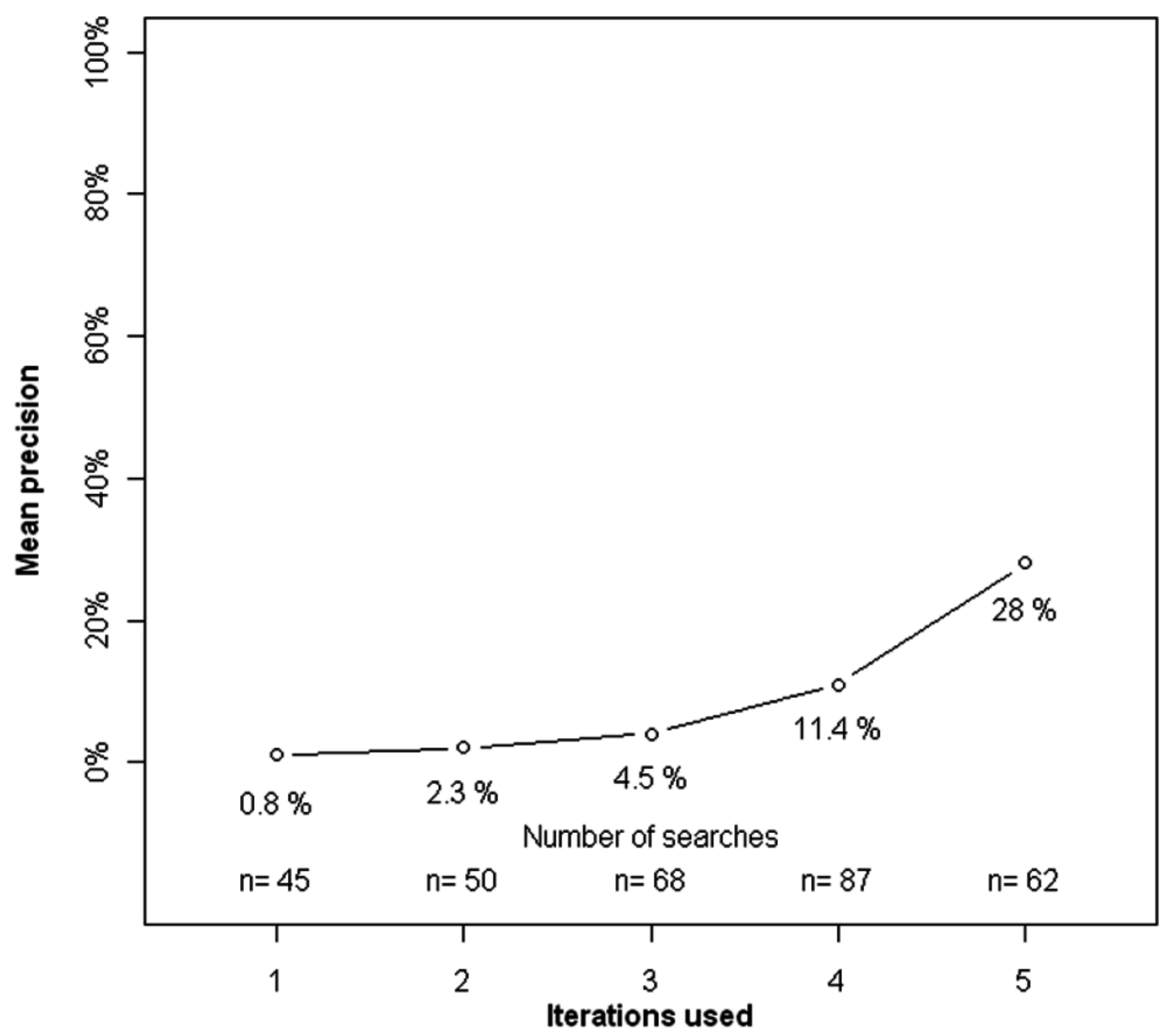


Table 3(on next page)

Table 3. Comparison of search strategies for retrieving high quality*, relevant PubMed citations

High quality citations were those reviewed by an evidence-based synoptic journal or accompanied by an editorial 
2 Table 3. Comparison of search strategies for retrieving high quality*, relevant PubMed citations.

\begin{tabular}{|c|c|c|c|c|}
\hline \multirow[t]{2}{*}{ Experimental } & \multicolumn{2}{|c|}{$\begin{array}{c}\text { PubMed Clinical Queries for } \\
\text { therapies }\end{array}$} & \multirow[t]{2}{*}{ Google } & \multirow[t]{2}{*}{$\begin{array}{l}\text { Google } \\
\text { Scholar }\end{array}$} \\
\hline & narrow & broad & & \\
\hline \multicolumn{5}{|c|}{$\begin{array}{l}\text { Precision of searches, median } \\
\text { (interquartile range) } \dagger+\end{array}$} \\
\hline $5.5 \% \S$ & $4.0 \% \S$ & $2.0 \%$ & $0 \% \S$ & $0 \% \S$ \\
\hline$(0 \%$ to $12 \%)$ & $(0 \%$ to $10 \%)$ & $(0 \%$ to $8 \%)$ & $(0 \%$ to $7 \%)$ & $(0 \%$ to $0 \%)$ \\
\hline \multicolumn{5}{|c|}{$\begin{array}{l}\text { Number of citations retrieved, median } \\
\text { (interquartile range) }+\dagger\end{array}$} \\
\hline $\begin{array}{c}2 \S \\
(0 \text { to } 6)\end{array}$ & $\begin{array}{c}2 \\
(0 \text { to } 4)\end{array}$ & $\begin{array}{c}1 \\
(0 \text { to } 3)\end{array}$ & $\begin{array}{c}0 \S \\
(0 \text { to } 2)\end{array}$ & $\begin{array}{c}0 \S \\
(0 \text { to } 0)\end{array}$ \\
\hline \multicolumn{5}{|c|}{$\begin{array}{l}\text { Proportion of searches that retrieved at least one citation } \\
\qquad(95 \% \text { confidence intervals }) \dagger\end{array}$} \\
\hline $\begin{array}{c}73 \% \S \\
(68 \% \text { to } 78 \%)\end{array}$ & $\begin{array}{c}63 \% \\
(58 \% \text { to } 68 \%)\end{array}$ & $\begin{array}{c}65 \% \\
(59 \% \text { to } 70 \%)\end{array}$ & $\begin{array}{c}46 \% \S \\
(41 \% \text { to } 52 \%)\end{array}$ & $\begin{array}{c}20 \% \S \\
(15 \% \text { to } 24 \%)\end{array}$ \\
\hline
\end{tabular}

3 * High quality citations were those reviewed by an evidence-based synoptic journal or accompanied by an

4 editorial.

$5 \uparrow \mathrm{P}<0.001$ for differences among groups.

$6 \$$ Note that rank sums can differ significantly although medians are the same.

$7 \S \mathrm{P}<0.05$ for difference compared to other groups.

8 Search results were limited to a maximum of 50 per search.

9 